\title{
Computer-guided porous materials design: from rationalization to prediction
}

\author{
Angeles Pulido ${ }^{1}$, Anna G. Slater ${ }^{2}$, Linjiang Chen², Marc A. Little ${ }^{2}$, Samantha Y. Chong ${ }^{2}$, Dan \\ Holden ${ }^{2}$, Tomasz Kaczorowski ${ }^{2}$, Ben J. Slater ${ }^{2}$, David P. McMahon ${ }^{1}$, Andrew I. Cooper ${ }^{2}$, \\ Graeme M. Day ${ }^{1}$ \\ ${ }^{1}$ Department of Chemistry, University of Southampton, Southampton, U. K.; ${ }^{2}$ Department of \\ Chemistry, University of Liverpool, Liverpool, U. K. \\ E-mail: M.Junquera-Pulido@soton.ac.uk
}

Organic molecules will tend to pack in dense crystal structures, avoiding the formation of voids. However, the generally less energetically favourable arrangement of molecules into porous crystal structures show important advantages in applications such as gas storage, separation or catalysis. Weak -electrostatic and dispersive- intermolecular interactions dominate molecular packing and are the origin of the unpredictability that seems to surround their crystallization. Thus, the rational design of new materials for technological applications will be limited by the ability to reliably anticipate: (i) the final crystal structure formed and (ii) the physico-chemical properties of such a material.

In this contribution we show how computational techniques and, in particular, crystal structure prediction (CSP) can be successfully used to get a better understanding of the crystal packing preferences of covalently bonded tubular porous organic cages (over 200 atoms); which could be only partially understood experimentally. CSP was used to show how small structural modifications of the molecular cages lead to drastic modifications of crystal packing preferences and help rationalize experimental crystallization outcomes ${ }^{[1]}$.

Moreover, it will be shown how CSP enabled the identification of a highly porous organic molecular crystal, with the lowest crystal density reported so far; which was subsequently synthesized ${ }^{[2]}$. It will be discuss how using the molecular diagram as the only input, CSP and property prediction were combined to build energy-structure-function (ESF) maps (see Scheme 1), and used to reveal the potential of materials for methane storage or alkane separation, which were experimentally confirmed. The potential of ESF maps for computer-guided crystal engineering will be also discussed.

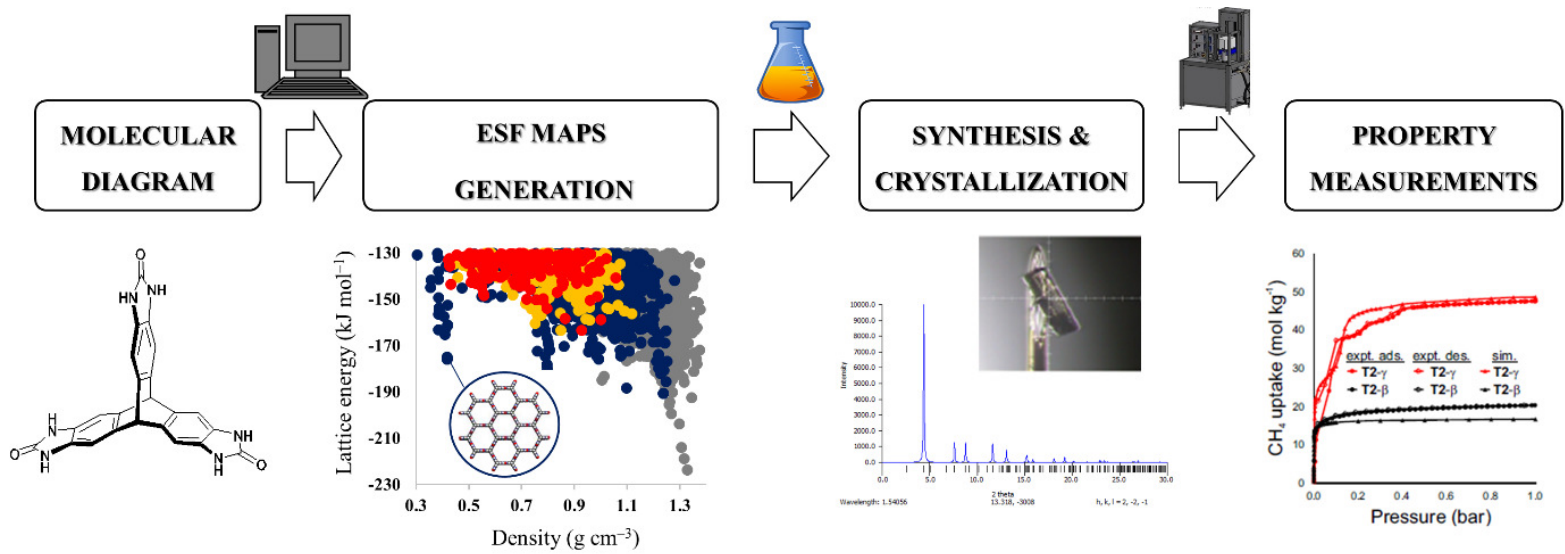

Scheme 1. Representation of the strategy used for computer guided organic molecular crystals design.

\section{References.}

[1] A. G. Slater, et al., Nat. Chem. 2017, 9, 17. [2] A. Pulido, et. al., Nature, accepted, 2017. 